\title{
DETECÇÃO DE EVENTOS ADVERSOS RELACIONADOS A MEDICAMENTOS POR MEIO DAS FERRAMENTAS GLOBAL TRIGGER TOOL E MEDICATION SAFETY THERMOMETER: UMA REVISÃO NARRATIVA
}

\section{DETECTION OF ADVERSE DRUG EVENTS USING THE GLOBAL TRIGgER TOOL AND THE MEDICATION SAFETY THERMOMETER: A NARRATIVE REVIEW}

\author{
Priscila Bernardi Garzella1, Denise Bueno ${ }^{2}$, Isabela Heineck ${ }^{1,2}$
}

\begin{abstract}
RESUMO
Eventos adversos (EA) são incidentes que resultam em dano ao paciente e representam um problema global. Estima-se que um em cada 10 pacientes sofram algum tipo de EA durante sua hospitalização. EA relacionados a medicamentos são a segunda causa mais frequente e a maior causa de danos evitáveis causados aos pacientes mundialmente. Esforços no sentido de evitá-los são de extrema importância, para tanto, é indispensável a mensuração dos eventos e danos associados. Ferramentas como Global Trigger Tool (GTT) e Medication Safety Thermometer (MedST) vêm sendo utilizadas para identificação e mensuração de EA relacionados a medicamentos, visando projeção de sistemas de saúde mais seguros. Este trabalho consiste em uma revisão narrativa da literatura, de caráter descritivo com enfoque nas vantagens e limitações das ferramentas GTT e MedST, vislumbrando contribuir com a literatura acerca da segurança no uso de medicamentos e encorajar o desenvolvimento e/ou adaptação transcultural e validação de ferramentas com esta finalidade para uso no Brasil. A ferramenta GTT possui caráter retrospectivo, o que inviabiliza ações preventivas e está limitada a apenas uma fonte de dados, o prontuário do paciente, diferente da MedST, que permite a mensuração prospectiva, possibilitando intervenções imediatas e busca das informações em diferentes fontes de dados.
\end{abstract}

Palavras-chave: Erros de medicação; segurança do paciente; Indicadores básicos de saúde

\begin{abstract}
Adverse events $(A E)$ are incidents that result in harm to the patient and represent a global problem. Estimates show that one in 10 patients experience some type of $A E$ during hospitalization. Adverse drug events are the second most common cause and the biggest cause of preventable harm to patients worldwide. Efforts to avoid them are extremely important; therefore, measurement of events and associated harms is essential. Tools such as the Global Trigger Tool (GTT) and the Medication Safety Thermometer (MedST) have been increasingly used for the identification and measurement of adverse drug events, aiming at the design of safer health systems. This paper consists of a narrative review of the literature focusing on the description of advantages and limitations of both the GTT and the MedST. The aim is to contribute to the literature on safer use of medications and to encourage the development and/or cross-cultural adaptation and validation of adequate tools for use in Brazil. The GTT is a retrospective tool, hindering preventive actions, and is limited to only one data source, i.e., the patient's medical record. Conversely, the MedST provides prospective measurement, allowing immediate interventions, and search for information in different data sources.
\end{abstract}

Keywords: Medication errors; patient safety; health status indicators
Clin Biomed Res. 2020;40(4):235-241

1 Departamento de Pós-Graduação em Ciências Farmacêuticas, Universidade Federal do Rio Grande do Sul. Porto Alegre, RS, Brasil.

2 Departamento de Pós-Graduação em Assistência Farmacêutica, Universidade Federal do Rio Grande do Sul. Porto Alegre, RS, Brasil.

Autor correspondente: Priscila Bernardi Garzella priscila.bernardi@hotmail.com Departamento de Pós-Graduação em Ciências Farmacêuticas, Universidade Federal do Rio Grande do Sul. Avenida Ipiranga, 2752, Sala 101 90610-000, Porto Alegre, RS, Brasil. 


\section{INTRODUÇÃO}

Eventos adversos (EA) são incidentes que resultam em dano ao paciente e representam um problema de saúde global ${ }^{1}$. De acordo com a Organização Mundial da Saúde, (OMS), estima-se que 1 em cada 10 pacientes hospitalizados sofram algum tipo de $E^{2}$. Erros relacionados a medicamentos, definidos como: "qualquer evento evitável nos processos de prescrição, transcrição, dispensação, administração ou monitorização do medicamento, que pode causar ou conduzir à utilização inadequada do medicamento ou dano ao paciente enquanto o medicamento está sob controle do profissional de saúde, paciente ou consumidor" 1 , são responsáveis por cerca de 18,3\% dos EA relatados ${ }^{2}$. Eventos decorrentes de erros de medicação são frequentemente evitáveis e estão associados a custos adicionais aos serviços de saúde e aumento no tempo de permanência dos pacientes internados ${ }^{3}$.

Reduzir os danos ocasionados por medicamentos tornou-se uma prioridade global através do Terceiro Desafio de Segurança do Paciente lançado pela OMS em $2017^{4}$. Este desafio objetiva reduzir em $50 \%$ os danos graves e evitáveis relacionados a medicamentos, até 2022 , através, dentre outras ações, da avaliação da natureza e o escopo dos danos evitáveis e fortalecimento dos sistemas de monitoramento para detectar e rastrear esses danos ${ }^{4}$.

Alinhado ao objetivo do Terceiro Desafio, ferramentas estruturadas como Global Trigger Tool (GTT) ${ }^{5,6}$ e Medication Safety Thermometer (MedST) ${ }^{7}$ vêm sendo utilizadas para identificação e mensuração de EA relacionados a medicamentos no ambiente hospitalar, tornando possível a compreensão da magnitude do problema visando projeção de sistemas de saúde mais seguros ${ }^{8}$. A ferramenta GTT foi desenvolvido pelo Institute for Healthcare Improvement $(\mathrm{IHI})^{5,6} \mathrm{em}$ 2004 como uma ferramenta de melhoria de qualidade e, desde então, vem sendo utilizada na prática clínica para identificar, rastrear e estimar taxas de EA, incluindo os eventos relacionados a medicamentos ${ }^{9}$. MedST é uma ferramenta desenvolvida pelo National Health Service $(\mathrm{NHS})^{7}$, em 2013, como parte de um programa de melhoria nacional que objetiva avaliar a segurança no uso de medicamentos em pacientes internados e vem sendo utilizada em mais de 100 instituições de saúde no Reino Unido ${ }^{10}$.

Ambas as ferramentas permitem identificar EA relacionados a medicamentos em ambiente hospitalar e foram desenvolvidas por reconhecidas agências internacionais ${ }^{5-7}$. Considerando que não possuímos no Brasil nenhuma ferramenta desenvolvida e validada que permita este tipo de mensuração, esta revisão visa explorar vantagens e limitações, bem como a aplicabilidade destas ferramentas de medidas, vislumbrando contribuir com a literatura acerca da segurança no uso de medicamentos e encorajar o desenvolvimento e/ou adaptação transcultural e validação de ferramentas com esta finalidade para uso no contexto brasileiro.

\section{MÉTODOS}

Trata-se de uma revisão narrativa da literatura de caráter descritivo, com enfoque nas metodologias GTT e MedST. A identificação e a seleção dos artigos foram realizadas no período de fevereiro a abril de 2020 de forma independente por duas pesquisadoras capacitadas, nas seguintes bases de dados: Nacional Library of Medicine (PubMed), Scientific Eletronic Library Online (SciELO), Medline e Biblioteca Virtual em Saúde do Ministério da Saúde (BVS) utilizando os termos: Medication Safety Thermometer, Global Trigger Tool e eventos adversos relacionados a medicamentos/adverse drug events. A revisão teve como abordagem os aspectos mais relevantes relacionados às metodologias em questão, com foco em suas vantagens e desvantagens.

Foram incluídos na pesquisa artigos originais e artigos de revisão que se enquadram na temática estabelecida, publicados entre 2008 e 2020, disponíveis com texto completo e publicados em periódicos nacionais e internacionais nos idiomas inglês ou português. As referências dos artigos selecionados foram verificadas a fim de identificar outros artigos que atendessem aos critérios de inclusão e que não estivessem captados ao fim da busca inicial. Os desacordos referentes a identificação e seleção de artigos entre as pesquisadoras foram resolvidos por consenso. Utilizou-se como critérios de exclusão: artigos que não estavam disponíveis eletronicamente na íntegra e artigos duplicados.

Foi realizada leitura exploratória dos artigos selecionados inicialmente seguida de leitura seletiva a fim de identificar àqueles que atendessem ao objetivo do estudo. Nos estudos incluídos nesta revisão, foi realizada leitura analítica e por fim leitura interpretativa e redação.

\section{RESULTADOS E DISCUSSÃO}

Através da pesquisa nas bases de dados selecionadas foram identificados 50 artigos e destes, 9 foram excluídos após a leitura do título. Foram avaliados os resumos dos 41 artigos restantes e, após esta etapa, 28 artigos foram selecionados para leitura do texto na íntegra. Quatro artigos foram incluídos a partir de análise da lista de referências dos estudos. Ao final desta etapa 18 artigos foram incluídos na revisão narrativa, conforme Figura 1. 


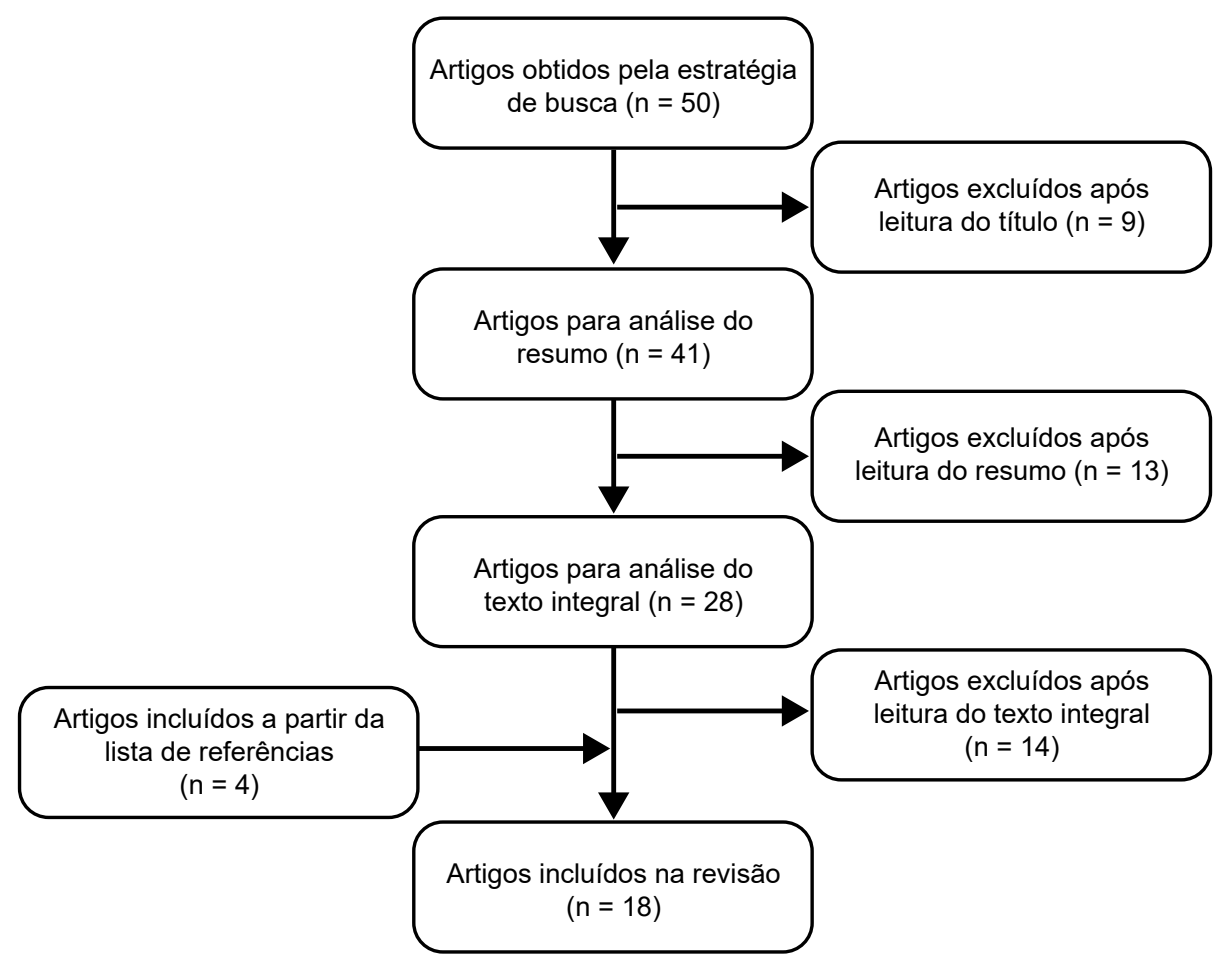

Figura 1: Diagrama de fluxo da revisão narrativa.

\section{Global Trigger Tool}

A ferramenta GTT foi desenvolvida pelo IHI como uma alternativa às tentativas convencionais de mensurar $E A^{5,6}$. A metodologia baseia-se na identificação de sinais de EA relacionados à prestação ativa de cuidado, ou seja, cuidados que não foram realizados (omitidos) e desencadearam dano ao paciente não são considerados ${ }^{11,12}$. Por exemplo, um paciente que não foi tratado adequadamente para hipertensão e sofreu um derrame por cuidados inadequados não é considerado no GTT pois o evento foi causado por omissão de cuidado. No entanto, um paciente que recebeu anticoagulante e posteriormente sofreu um acidente vascular cerebral devido a hemorragia intracraniana, é considerado um EA analisado pelo GTT pois foi desencadeado por um cuidado ativo ${ }^{6}$.

$O$ conceito de EA adotado nesta metodologia é: "danos físicos ocasionados ou agravados por cuidados da equipe interprofissional que requeiram monitoramento, tratamento ou hospitalização, ou que resulte em morte" e estes são categorizados seguindo as definições do National Coordinating Council for Medication Error Reporting and Prevention (NCC MERP) 6 . Um sinal indica a possibilidade de ocorrência de EA e a necessidade de avaliação para confirmação ${ }^{12}$. Esta ferramenta consiste na mensuração de EA através da revisão retrospectiva de prontuários identificando os sinais divididos em seis módulos: cuidados, medicação, cirurgia, terapia intensiva, perinatal e emergência ${ }^{6}$.

Em relação a medicamentos, o GTT busca a identificação de EA através de treze sinais, cada um deles identificado por meio de um código que inicia com a letra $\mathrm{M}$ referindo-se a medicamentos e numerados na sequência, conforme descrito na Tabela $1^{6,13}$.

Tabela 1: Sinais de EA relacionados a medicamentos utilizados na ferramenta Global Trigger Tool.

\begin{tabular}{cl}
\hline Código & \multicolumn{1}{c}{ Sinal } \\
\hline M 01 & Cultura de Clostridium difficile positiva \\
M 02 & TTPa > 100 segundos \\
M 03 & RNI>6 \\
M 04 & Glicose < 50 mg/dL \\
M 05 & Ureia em ascensão ou creatinina sérica \\
& duas vezes (2x) o valor inicial \\
M 06 & Administração de vitamina K \\
M 07 & Administração de difenidramina \\
M 08 & Administração de flumazenil \\
M 09 & Administração de naloxona \\
M 10 & Administração de antiemético \\
M 11 & Sedação excessiva e hipotensão \\
M 12 & Interrupção abrupta de medicamento \\
M 13 & Outros \\
\hline
\end{tabular}

TTPA: Tempo de tromboplastina parcialmente ativada; RNI: Razão normalizada internacional; M: sinal referente a seção de medicamentos. 
Os indicadores relacionados a medicamentos mensurados com o GTT são: eventos adversos por 1000 pacientes-dia; eventos adversos por 100 admissões e percentual de internações com eventos adversos. "Eventos adversos por 1000 pacientes-dia" é uma medida tradicional recomendada para monitorar a taxa de danos ao longo do tempo, além de fornecer uma representação mais fácil da taxa de danos para lideranças. Os indicadores citados são classificados como de resultado. Para subsidiar medidas de melhoria, além de indicadores de resultado é recomendada a mensuração de indicadores de processo. Estes permitem identificar processos frágeis que, se melhorados, impactarão diretamente no resultado. No conjunto de dados mensurados através do GTT, não estão descritos indicadores de processo, o que torna dificultoso os esforços de melhoria ${ }^{6}$.

A revisão do prontuário para a coleta de dados deve ser realizada por uma equipe multiprofissional de no mínimo 3 pessoas, incluindo um médico que irá definir quais os sinais que resultaram em dano. A equipe deve ser capacitada e possuir habilidade para identificar os sinais no tempo proposto pela metodologia ${ }^{6}$. Cabe citar que a substituição da equipe só deve ser realizada se for de extrema importância e, com capacitação adequada dos novos profissionais a fim de não comprometer os dados ${ }^{14}$. A aplicação da ferramenta GTT envolve diversas etapas, tornando o processo demorado: em torno de 20 minutos para cada um dos 10 prontuários, quinzenalmente ${ }^{13}$. Segundo estudo de Schildmeijer et al. ${ }^{12}$ que avaliou os pontos fortes e fracos do GTT através de entrevistas estruturadas com profissionais que fazem uso da ferramenta, uma das fraquezas, de acordo com os entrevistados, é o fato da ferramenta não capturar as falhas por omissão de cuidado que também estão relacionadas a EA. Além disso, os profissionais relataram que alguns sinais utilizados são imprecisos e outros nunca foram utilizados ${ }^{13}$.

A medição dos resultados de segurança é retrospectiva sendo útil para a aprendizagem, mas limitada para intervenções imediatas que impeçam o erro/dano, visto que, conforme recomendação da metodologia, os pacientes avaliados na amostra já receberam alta hospitalar no momento da coleta $^{15}$. Para envolver, educar e mobilizar a equipe de saúde que está na "linha de frente" para que sejam feitas alterações no local de atendimento são necessárias ação e dados "em tempo real" ${ }^{15}$. Sendo assim, um método eficaz de coletar dados de forma prospectiva sobre os danos dos EAé indispensável no atendimento ${ }^{15,16}$.

Carnevali et al. ${ }^{14}$ avaliaram o desempenho do módulo de medicamentos do GTT (13 sinais) na identificação de EA relacionados a medicamentos em comparação a ferramenta de sinais de EA relacionados a medicamentos modificada para o contexto belga (20 sinais). Para tal, uma amostra aleatória de 20 admissões de pacientes adultos foi selecionada mensalmente ao longo de um período de 12 meses e analisadas através das duas metodologias. Através do módulo de medicamentos do GTT foram encontrados $77 \%$ do total de EA relacionados a medicamentos, isto significa que em torno de $25 \%$ dos EA relacionados a medicamentos identificados pela ferramenta modificada não são detectados pelo módulo de medicamentos do GTT, indicando a necessidade de refinamento dos sinais descritos no GTT para melhorar seu desempenho.

Afim de sanar com as limitações do GTT relacionadas ao elevado consumo de recursos de tempo e material humano, que limita seu uso, Mevik et al. ${ }^{17}$ modificaram o GTT tornando a busca ativa digital para identificar automaticamente os sinais nos registros eletrônicos de saúde (prontuários dos pacientes). Através de um estudo transversal exploratório, os autores compararam o GTT modificado com o GTT original. Foram incluídos no estudo 1233 registros selecionados entre março e dezembro de 2013 em um hospital norueguês de médio porte. Ambos os métodos identificaram 35 eventos adversos por 1000 pacientes-dia, não demonstrando diferença significativa na taxa de EA identificados pelos métodos ao longo do tempo. Em relação ao tempo total de revisão, através do GTT modificado 1233 registros foram revisados em um total de 23 horas, enquanto o tempo de revisão manual usando o método GTT original foi de 411 horas. Como resultados, os autores afirmam que o método GTT modificado é válido, confiável e eficiente para monitorar a taxa de EA e cumpriu seu propósito de diminuição de tempo comparado ao GTT original17-19.

Devido as várias etapas necessárias para identificação de EA utilizando o GTT, são necessários 20 minutos para rastrear o registro de 1 único paciente e há a exigência de um médico e de um farmacêutico na revisão, o que onera a equipe em termos de tempo e recursos ${ }^{7}$. Singh et al. ${ }^{20}$ avaliaram o desempenho do GTT em identificar EA relacionados a medicamentos entre idosos atendidos em ambulatórios de atenção primária. Dos 1289 registros revisados, 645 apresentaram pelo menos um sinal relacionado a evento adverso. Nove dos 39 sinais pesquisados foram responsáveis por $94,4 \%$ de todos os $E A$ relacionados a medicamentos detectados, sugerindo um refinamento dos sinais da ferramenta. Diante do exposto, torna-se necessário o aprimoramento do GTT para melhorar seu desempenho, fornecer dados em tempo real, ser exequível no ambiente de cuidados de saúde e mais eficiente em termos de tempo e recursos humanos ${ }^{7}$. 


\section{Medication Safety Thermometer}

O NHS criou, em 2010, como parte de um programa de melhoria da qualidade, a ferramenta NHS Safety Thermometer, que tem por objetivo fornecer a prevalência instantânea dos EA, através da mensuração de danos no foco do cuidado ao paciente ${ }^{10}$. Trata-se de um programa que inclui um conjunto de ferramentas de mensuração de prevalência de EA, chamadas de Classic Safety Thermometer, Mental Health Safety Thermometer, Maternity Safety Thermometer e Children \& Young People Safety Thermometer, relacionadas individualmente aos mais diversos objetivos, como quedas, lesão por pressão, tromboembolismo venoso, infecção o trato urinário, maternidade, saúde mental, crianças e idosos e medicamentos $7,10,15,21-24$.

A versão do Safety Thermometer destinada a mensuração da prevalência de EA relacionados a medicamentos foi desenvolvida em 2013 e chamase Medication Safety Thermometer ${ }^{7}$. O processo de desenvolvimento desta ferramenta foi descrito por Rostami et al. ${ }^{7} \mathrm{e}$, por se tratar de uma nova ferramenta e uma nova forma de mensurar dados relacionados a medicamentos, objetivando melhorias, a versão inicial do MedST foi testada durante nove meses. O processo de teste foi aprimorado através da utilização de ciclos Plan-Do-Study-Act (PDSA) e desde então, a ferramenta já sofreu 16 atualizações.

O MedST foi desenvolvido para apoiar as organizações de saúde no monitoramento dos erros relacionados a medicamentos e foi projetado para mensurar a melhoria ao longo do tempo ${ }^{7}$. A ferramenta é dividida em três etapas e identifica danos potenciais e reais. A etapa 1 identifica erros relacionados ao processo (omissão de dose, status de alergia e conciliação medicamentosa) que podem indicar potencial para dano. A segunda etapa só deve ser preenchida se for identificado na etapa 1 que o paciente recebeu algum medicamento considerado potencialmente perigoso (anticoagulantes, opioides, sedativo injetáveis e/ou insulina) nas últimas $24 \mathrm{~h}$. Nesta etapa os profissionais identificam os sinais de dano. A terceira etapa investiga o dano real e é realizada apenas se algum sinal for encontrado durante a etapa 2. Esta fase envolve equipe interprofissional (composta por no mínimo, um médico, um farmacêutico e um enfermeiro) que irá avaliar, a partir do sinal identificado, se houve ou não um dano ao paciente ${ }^{7}$.

A coleta de dados deve ser realizada in locu, no ambiente clínico, uma vez ao mês, incluindo $100 \%$ dos pacientes internados na instituição ${ }^{7}$. No entanto, é possível iniciar em uma escala menor escolhendo, por exemplo, uma enfermaria como piloto e ir evoluindo aos poucos ${ }^{7}$. As informações devem ser coletadas através de prontuários incluindo as prescrições médicas, de entrevistas com pacientes, cuidadores e profissionais de saúde e devem ser revisadas e utilizadas pelas organizações de saúde, de forma local ou agregando dados nacionais, para obtenção instantânea do nível de dano causado por medicamentos ${ }^{7}$.

Os indicadores mensurados através da aplicação da ferramenta são ${ }^{24}$ : proporção de pacientes com conciliação iniciada dentro das primeiras $24 \mathrm{~h}$ da admissão; proporção de pacientes com dose omitida nas últimas $24 \mathrm{~h}$; proporção de pacientes com status de alergia medicamentosa documentado; proporção de pacientes com omissão de dose de medicamentos crítico; proporção de pacientes que receberam medicamento potencialmente perigoso nas últimas $24 \mathrm{~h}$ e proporção de pacientes com sinal para dano. $\mathrm{O}$ MedST coleta dados relacionados a processos com potencial para erros e danos, sendo eles: conciliação medicamentosa, status de alergia, omissão de medicamentos, omissão de medicamento críticos e identificação de dano causado por medicamentos potencialmente perigosos ${ }^{7}$. Os dados coletados podem ser utilizados para construção de uma linha de base, direcionando os esforços de melhoria para então, mensurar as melhorias ao longo do tempo ${ }^{7}$.

No período de dezembro de 2015 a setembro de 2016, foi realizado um estudo no qual entrevistas semiestruturadas foram conduzidas com a linha de frente que implementou a ferramenta em unidades de atenção primária e secundária em saúde do NHS, avaliando o processo de implementação da ferramenta MedST. Foram realizadas 15 entrevistas estruturadas em três eixos: engajamento com o objetivo, coleta de dados e uso dos dados. As informações obtidas nas entrevistas foram interpretadas por meio de análise temática inicial seguida de análise pela Teoria do Processo de Normalização. De acordo com as informações coletadas nas entrevistas, os profissionais entenderam o objetivo da ferramenta e pode-se concluir que a mesma é intuitiva, acessível e capaz de ser aplicada por profissionais da saúde em menos de 10 minutos por paciente, com treino mínimo. Foi observada falta de engajamento da equipe em situações onde a liderança local não demonstrou apoio ao uso da ferramenta, sugerindo que o envolvimento do gestor é fundamental para alcançar o objetivo proposto e melhorar a segurança no uso de medicamentos. Outro achado importante deste estudo é a dificuldade de implementação da ferramenta para coleta de dados na atenção primária em comparação ao ambiente hospitalar, embasando a decisão da não aplicabilidade do MedST neste contexto ${ }^{23}$.

Por meio de estudo qualitativo realizado em três hospitais do Reino Unido, Rostami e colaboradores $(2019)^{22}$ buscaram descrever através de entrevistas semiestruturadas utilizando a Teoria do Processo de Normalização, se e como os dados coletados pelas equipes utilizando o MedST estavam sendo 
utilizados nas instituições para apoiar melhorias de segurança no uso de medicamentos. Dados coletados em três hospitais do NHS, entre outubro de 2013 e julho de 2016, foram analisados através de gráficos de tendência e as mudanças identificadas foram discutidas com as equipes que estavam utilizando a ferramenta. Os entrevistados demostraram confiar nos dados coletados e os utilizaram para direcionar os esforços de melhoria. De acordo com as informações obtidas nas entrevistas, o estudo evidenciou que, por se tratar de uma nova ferramenta, as equipes precisam de um tempo de aprendizado para a coleta e uso de dados para posteriormente, entender o que os resultados estão representando. A simples coleta de dados por si só não gera mudanças e melhorias nos sistemas, os dados coletados precisam ser revisados, analisados e monitorados ao longo do tempo em paralelo a implementação de mudanças e de processos mais seguros.

Rostami et al. ${ }^{21}$ determinaram a prevalência, natureza e fatores de omissão de medicamentos em pacientes através da compilação dos dados coletados em 37 hospitais que utilizaram o MedST, em janeiro de 2015. A prevalência de omissão de medicamentos encontrada na amostra foi de $30 \%$ e a recusa pelo paciente foi a causa de $50 \%$ das omissões. Dentre os fatores de risco, a polimedicação foi a principal causa: pacientes que possuíam mais de 20 medicamentos prescritos apresentaram chance 5 vezes maior de sofrerem omissão. O estudo ressalta que os hospitais utilizam os dados de omissão obtidos com o MedST juntamente com outros dados de mensuração, para iniciativas de melhoria no âmbito hospitalar. Neste estudo, os dados foram devolvidos à equipe juntamente com sugestões de estratégias de aprimoramento.

Diante do exposto, entende-se que a ferramenta MedST possui melhor desempenho, pois sua aplicação é mais rápida, o que faz com que haja mais tempo remanescente para a ação. Como supracitado, "a melhoria requer ação e dados em tempo real" para que a equipe seja mobilizada e sejam feitas mudanças no cuidado de forma eficiente. Além disto, a ferramenta MedST apresenta como vantagens o caráter prospectivo, através de identificação de danos potenciais por meio de medidas de processo, que permite intervenção de forma imediata evitando que o mesmo se torne um dano real ao paciente.

A ferramenta GTT possui caráter retrospectivo, o que inviabiliza a interferência imediata para evitar o dano. Também está limitada a apenas uma fonte de dados, o prontuário do paciente, que geralmente não reflete a prática clínica ${ }^{25}$. A outra ferramenta citada neste trabalho (MedST), por outro lado, permite utilizar além do prontuário, a equipe de saúde e o paciente como fontes de dados. As principais caraterísticas de cada uma das metodologias estão resumidas na Tabela 2.

Tabela 2: Principais características das ferramentas Global trigger tool e Medication Safety Thermometer.

\begin{tabular}{|c|c|c|}
\hline & Global Trigger Tool & Medication Safety Thermometer \\
\hline Tamanho da amostra & 10 pacientes & $100 \%$ dos pacientes internados na unidade/instituição \\
\hline Frequência de coleta & Quinzenal & Mensal \\
\hline Fonte de dados & $\begin{array}{l}\text { Prontuário do paciente, } \\
\text { incluindo prescrição médica }\end{array}$ & $\begin{array}{l}\text { Prontuário do paciente, incluindo prescrição médica } \\
\text { Entrevista com paciente } \\
\text { Entrevista com cuidador } \\
\text { Entrevista com equipe do cuidado }\end{array}$ \\
\hline Observação & Retrospectiva & Prospectiva \\
\hline $\begin{array}{l}\text { Tempo destinado a coleta de } \\
\text { dados por prontuário }\end{array}$ & 20 minutos & Sem tempo estipulado (até completar o formulário) \\
\hline $\begin{array}{l}\text { Número de sinais a serem } \\
\text { investigados }\end{array}$ & 13 & 16 \\
\hline Número de indicadores obtidos & 03 & 06 \\
\hline Validado para uso no Brasil & Não & Não \\
\hline
\end{tabular}

\section{CONCLUSÃO}

A forma de coleta é o diferencial entre as ferramentas. Considerando que o GTT rastreia EA de pacientes que já receberam alta hospitalar, não é possível evitar os danos, diferentemente da mensuração prospectiva da ferramenta MedST que possibilita identificar e evitar EA relacionados a medicamentos, reduzindo os danos e aumentando a segurança dos pacientes. As vantagens apresentadas em relação a ferramenta MedST encorajam o processo de adaptação transcultural e validação desta ferramenta para utilização no contexto brasileiro.

\section{Conflitos de Interesse}

Os autores declaram não haver conflitos de interesse. 


\section{REFERÊNCIAS}

1. World Health Organization. Conceptual Framework for the International Classification for Patient Safety. Version 1.1. Final Technical Report, January 2009 [Internet]. Geneva: WHO; 2009 [cited 2020 Apr 21]. Available from: https://www.who.int/patientsafety/ taxonomy/icps_full_report.pdf

2. World Health Organization. Patient Safety. Making health care safer [Internet]. Geneva: WHO; 2017 [cited 2020 Apr 21]. Available from: https://apps.who.int/iris/ bitstream/handle/10665/255507/ WHO-HIS-SDS-2017.11-eng. pdf? sequence=1\&isAllowed $=y$

3. Prevalence and cost of medication errors in England. Drug Ther Bull [Internet]. 2018 [cited 2020 Mar 3];56(8):89. Available from: http:// dx.doi.org/10.1136/dtb.2018.8.0656

4. Donaldson LJ, Kelley ET, Dhingra-Kumar N, Kieny M, Sheikh A. Medication Without Harm: WHO's Third Global Patient Safety Challenge. Lancet [Internet]. 2017 [cited 2020 Mar 3];389(10080):1680-1. Available from: http://dx.doi.org/10.1016/ s0140-6736(17)31047-4

5. Griffin FA, Resar RK. IHI Global Trigger Tool for Measuring Adverse Events. IHI Innovation Series white paper. Cambridge, MA: Institute for Healthcare Improvement; 2007.

6. Griffin FA, Resar RK. IHI Global Trigger Tool for Measuring Adverse Events. IHI Innovation Series white paper. 2a ed. Cambridge, MA: Institute for Healthcare Improvement; 2009.

7. Rostami P, Power M, Harrison A, Bramfitt K, Williams SD, Jani Y, et al. Learning from the design, development and implementation of the Medication Safety Thermometer. Int J Qual Health Care. 2017;29(2):301-9.

8. Makary MA, Daniel M. Medical error: the third leading cause of death in the US. BMJ. 2016;353:i2139.

9. Hanskamp-Sebregts $M$, Zegers $M$, Vincent C, van Gurp PJ, de Vet HCW, Wollersheim H. Measurement of patient safety: a systematic review of the reliability and validity of adverse event detection with record review. BMJ Open. 2016;6(8):e011078.

10. Power M, Stewart K, Brotherton A. What is the NHS Safety Thermometer? Clin Risk [Internet]. 2012 [cited 2020 Mar 3]; 18(5): 163-9. Available from: http://dx.doi. org/10.1258/cr.2012.012038

11. Farup PG. Are measurements of patient safety culture and adverse events valid and reliable? Results from a cross sectional study. BMC Health Serv Res. 2015;15:186.

12. Schildmeijer K, Unbeck M, Muren O, Perk J, Härenstam KP, Nilsson L. Retrospective record review in proactive patient safety work: identification of no-harm incidents. BMC Health Serv Res. 2013;13:282.

13. Schildmeijer K, Nilsson L, Perk J, Arestedt K, Nilsson G. Strengths and weaknesses of working with the Global Trigger Tool method for retrospective record review: focus group interviews with team members. BMJ Open. 2013;3(9):e003131.

14. Carnevali L, Krug B, Amant F, Van Pee D, Gérard V, de Béthune X, et al. Performance of the adverse drug event trigger tool and the global trigger tool for identifying adverse drug events: experience in a Belgian hospital. Ann Pharmacother. 2013;47(11):1414-9.

15. Power M, Fogarty M, Madsen J, Fenton K, Stewart K, Brotherton A, et al. Learning from the design and development of the NHS Safety Thermometer. Int J Qual Health Care. 2014;26(3):287-97.

16. Hibbert PD, Molloy CJ, Hooper TD, Wiles LK, Runciman WB, Lachman P, et al. The application of the Global Trigger Tool: a systematic review. Int J Qual Health Care. 2016;28(6):640-9.

17. Mevik K, Hansen TE, Deilkås EC, Ringdal AM, Vonen B. Is a modified Global Trigger Tool method using automatic trigger identification valid when measuring adverse events? Int $J$ Qual Health Care. 2019;31(7):535-40.
18. Jick H. Drugs: Remarkably Nontoxic. N Engl J Med [Internet]. 1974 [cited 2020 Mar 3];291:824-8. Available from: http://dx.doi.org/10.1056/ nejm197410172911605

19. Classen DC, Pestotnik SL, Evans RS, Burke JP. Description of a computerized adverse drug event monitor using a hospital information system. Hosp Pharm. 1992;27(9):774, 776-9, 783.

20. Singh $R$, McLean-Plunckett EA, Kee R, Wisniewski A, Cadzow R, Okazaki S, et al. Experience with a trigger tool for identifying adverse drug events among older adults in ambulatory primary care. Qual SafHealth Care. 2009;18(3):199-204.

21. Rostami $P$, Heal $C$, Harrison $A$, Parry G, Ashcroft DM, Tully MP. Prevalence, nature and risk factors for medication administration omissions in English NHS hospital inpatients: a retrospective multicenter study using Medication Safety Thermometer data. BMJ Open. 2019;9(6):e028170.

22. Rostami P, Harrison A, Parry G, Ashcroft D, Tully MP. A qualitative study exploring how routinely collected Medication Safety Thermometer data have been used for quality improvement purposes using case studies from three UK hospitals. BMJ Open. 2019;9(2):e025292.

23. Rostami P, Ashcroft DM, Tully MP. A formative evaluation of the implementation of a medication safety data collection tool in English healthcare settings: A qualitative interview study using normalisation process theory. PLoS One. 2018;13(2):e0192224.

24. Harm Free Care. Harm Free Care: Resources for patient harm reduction [Internet]. [cited 2020 Apr 19]. London: Harm Free Care; 2020. Available from: http://harmfreecare.org/wp-content/files mf/NHS-SafetyThermometer_V14.pdf

25. De Marinis MG, Piredda M, Pascarella MC, Vincenzi B, Spiga F, Tartaglini D, et al. 'If it is not recorded, it has not been done!'? consistency between nursing records and observed nursing care in an Italian hospital. J Clin Nurs. 2010; 19(11-12):1544-52. 